Ophthalmologe 2009 · 106:397-398

DOI 10.1007/s00347-009-1933-1

Online publiziert: 25. April 2009

(c) Springer Medizin Verlag 2009

\author{
C. Cursiefen \\ Universitätsaugenklinik, Friedrich-Alexander-Universität Erlangen-Nürnberg, Erlangen
}

\title{
Bevacizumab goes anterior
}

\section{Antiangiogene Therapie am vorderen Augenabschnitt}

Die Hornhaut als eines der wenigen avaskulären Gewebe des Menschen ist seit Jahrzehnten das In-vivo-Modell zum Studium der Mechanismen der Angiogenese (Blutgefäßentstehung). Dr. Judah Folkman, Begründer der Angiogeneseforschung, etablierte bereits in den 7oer Jahren Tiermodelle zur Entwicklung von Angiogenesehemmstoffen, in denen die Hornhaut eine zentrale Rolle spielte [1]. Aufbauend auf diesen Vorarbeiten wurden in den letzten Jahren zahlreiche endogene und exogene Hemmstoffe der pathologischen Gefäßneubildung identifiziert. Diese haben inzwischen in die Klinik Einzug gehalten und kommen routinemäßig z. B. bei Tumorpatienten und bei angiogenen Erkrankungen am Augenhinterabschnitt (AMD, diabetische Retinopathie etc.) zum Einsatz.

Im Gegensatz dazu hat sich die Angiogenesehemmung erst kürzlich wieder ihrem Ursprungsort, nämlich der Hornhaut, zugewandt. Es gibt am vorderen Augenabschnitt zahlreiche visusbedrohende und zur Erblindung führende Erkrankungen, die mit einer pathologischen Blutgefäßund/oder Lymphgefäßaussprossung (Angiogenese und Lymphangiogenese) assoziiert sind.

Ziel dieses Themenhefts ist es, Indikationen zur antiangiogenen Therapie am vorderen Augenabschnitt und aktuelle Möglichkeiten der lokalen Angiogenesehemmung darzustellen. Dies umfasst nicht nur die pathologische Gefäßeinsprossung in der Hornhaut, die per se $\mathrm{zu}$ einer deutlichen Sehverschlechterung bis hin zur Erblindung führt und mit dem Trachom und der herpetischen Keratopa- thie mit zu den häufigsten Erblindungsursachen weltweit gehört, sondern auch die Hornhautneovaskularisation im Kontext der Hornhauttransplantation. Pathologische Gefäßeinsprossungen in die Hornhaut sowohl vor als auch nach Keratoplastik, gehören zu den stärksten Risikofaktoren für eine Immunreaktion nach Keratoplastik $[2,3,4]$. Topische antiangiogene Therapie an der Hornhaut kann Transplantatüberleben verbessern. Dieses neue Therapiekonzept („Immunmodulation durch antiangiogene Therapie“) konnte im Tiermodell bereits als wirksam nachgewiesen werden $[2,3,4]$. Pathologische Neovaskularisationen der Hornhaut gefährden daneben den Erfolg von Limbusstammzelltransplantationen, worauf auch in dem Beitrag aus Erlangen eingegangen wird [5].

Neben der Hornhaut stellt die pathologische Gefäßneubildung im Rahmen der konjunktivalen Wundheilung beim Patienten nach filtrierender Glaukomchirurgie ein wichtiges klinisches Problem dar. Neue antiangiogene Therapieansätze können hier das längerfristige Funktionieren von Filterkissen nach Glaukomchirurgie sichern [6]. In der Arbeit von Grisanti et al. aus Lübeck [7] wird ausführlich der aktuelle Stand der antiangiogenen Therapie nach Glaukomchirurgie dargestellt. Ein weiterer Schwerpunkt dieses Artikels ist die Möglichkeit der antiangiogenen Therapie bei Rubeosis iridis und nachfolgenden neovaskulären Glaukomen.

Eine weitere wichtige Indikation zur antiangiogenen Therapie am vorderen Augenabschnitt stellt das rezidivierende Pterygium dar, in dessen Pathogenese eine überschießende Angiogenese eine wichtige Rolle spielt. In der Übersicht von Geerling et al. [8] aus Würzburg werden aktuelle Ansätze zur antiangiogenen Therapie beim Pterygium und in der Bindehaut erörtert. Des Weiteren konnten wir kürzlich zeigen, dass auch Tumoren im Bereich der Bindehaut (z. B. das maligne Melanom der Bindehaut) eine eigene Angiogenese und speziell auch Lymphangiogenese induzieren, über die ein erhöhtes Tumormetastasierungsrisiko vermittelt wird [9]. Auch diese Erkrankungen stellen eine Indikation für eine antiangiogene Therapie am vorderen Auge dar.

Dank der spektakulären Fortschritte in der Angiogeneseforschung sind wir inzwischen in der Lage, auch am vorderen Augenabschnitt eine lokale und spezifische antiangiogene Therapie durchzuführen. Dies ist ebenfalls ein wichtiges Thema dieses Themenhefts und zurzeit zum einen möglich mit dem Off-Label-Gebrauch von VEGF-Antikörpern wie z. B. Avastin ${ }^{\circledR}$ als Augentropfen (Bevacizumab), die inzwischen in mehreren Studien als relativ sicher und effektiv nachgewiesen wurden. Des Weiteren stehen mit Antisenseoligonukleotid-Augentropfen gegen Insulinrezeptorsubtrat1 (GS-101) spezifische antiangiogene Augentropfen zur Verfügung, die bereits in einer Phase-II-Studie erfolgreich getestet wurden und sich zur Zeit in der Phase III Studie bewähren müssen [10]. Damit besteht die Hoffnung, mittelfristig auch zugelassene Möglichkeiten zur spezifischen antiangiogenen Therapie an der Hornhaut, Bindehaut und am vorderem Augensegment zur Verfügung zu haben. Die 
Übersicht aus der Universitätsaugenklinik in Erlangen [5] stellt die aktuellen Möglichkeiten zur topischen antiangiogenen Therapie an der Hornhaut mit Avastin ${ }^{\bullet}$ Augentropfen und GS-101 Augentropfen dar und erörtert auch ihr Sicherheits- und Effektivitätsprofil [11, 12, 13].

Zusammenfassend kehrt die antiangiogene Therapie nach einem über 30-jährigen Weg wieder zu ihrem Ursprungsangriffsort, nämlich an die Hornhaut, zurück. Der antiangiogene Therapieansatz hat sich vom Krankenbett ins Labor und wieder zurück ans Krankenbett durchgesetzt, sodass wir auch an der Hornhaut, Bindehaut und Vorderkammer Möglichkeiten haben, spezifisch pathologische Gefäßaussprossungen, die Sehverschlechterungen mit sich bringen oder Transplantatüberleben bedrohen, therapieren zu können. Mit Avastin - und GS-101Augentropfen gibt es nun Möglichkeiten, spezifisch lokal antiangiogene Therapie zu betreiben. Zukünftige Studien müssen aber viele offene Fragen klären: So ist es derzeit noch unklar, in welcher Dosierung antiangiogene Augentropfen gegeben werden sollen, wie lange die Therapie benötigt wird und was man gegen etablierte mature Blut- und auch Lymphgefäße tun kann. Des Weiteren ist das langfristige Sicherheitsprofil der antiangiogenen Therapie am vorderen Augenabschnitt eine noch genauer zu untersuchende Frage.

Ihnen viel Freude bei der Lektüre der drei Übersichten zum Thema "Antiangiogene Therapie am vorderen Augenabschnitt" aus Erlangen, Lübeck und Würzburg, die auf ein gemeinsames Symposium auf der DOG 2008 in Berlin zurückgehen.

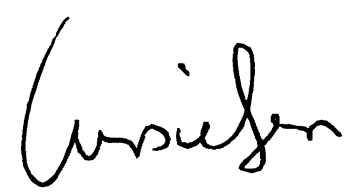

Claus Cursiefen

\section{Korrespondenzadresse PD Dr. C. Cursiefen}

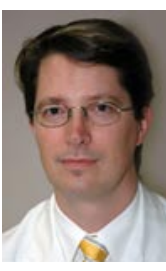

Universitätsaugenklinik,

Friedrich-Alexander-Universität

Erlangen-Nürnberg

Schwabachanlage 6

91054 Erlangen

ccursiefen@yahoo.com

Interessenkonflikt. Der korrespondierende Autor gibt an, dass kein Interessenkonflikt besteht.

\section{Literatur}

1. Regenfuss B, Bock F, Parthasarathy A, Cursiefen C (2008) Corneal (lymph)angiogenesis -from bedside to bench and back: a tribute to Judah Folkman. Lymphat Res Biol 6:191-201

2. Cursiefen C, Maruyama K, Liu Y et al (2004) Inhibition of hemangiogenesis and lymphangiogenesis after normal-risk corneal transplantation by neutralizing VEGF promotes graft survival. Invest Ophthalmol Vis Sci 45:2666-2673

3. Bachmann B, Bock F, Wiegand S et al (2008) VEGFA-neutralization after high-risk corneal transplantation promotes graft survival. Arch Ophthalmol 126:71-77

4. Bachmann B, Luetjen-Drecoll E, Bock F et al (2009) Transient postoperative VEGF-neutralisation improves graft survival in corneas with partly regressed inflammatory neovascularisation. $\mathrm{Br}$ J Ophthalmol (in press)

5. Regenfuss B, Bock F, Bachmann B et al (2009) Topische antiangiogene Therapie an der Hornhaut: Sicherheit und Effektivität. Ophthalmol 106:399406

6. Grewal DS, Jain R, Kumar H, Grewal SP (2008) Evaluation of subconjunctival bevacizumab as an adjunct to trabeculectomy a pilot study. Ophthalmology 115:2141-2145

7. Lüke J, Lüke M, Grisanti S (2009) Antiangiogene Therapie bei neovaskulärem Glaukom und nach Filterkissenchirurgie. Ophthalmol 106:407-412

8. Leippi S, Grehn F, Geerling G (2009) Antiangiogene Therapie bei Pterygium-Rezidiven. Ophthalmol 106:413-419

9. Heindl LM, Hofmann TN, Knorr HL et al (2009) Intraocular lymphangiogenesis in malignant melanomas of the ciliary body with extraocular extension. Invest Ophthalmol Vis Sci (Epub ahead of print)

10. Cursiefen C, Bock F, Horn FK et al (2009) GS-101 antisense oligonucleotide eye drops inhibit corneal neovascularization: interim results of a randomized phase II trial. Ophthalmology (in press)

11. Bock F, Onderka J, Dietrich T et al (2007) Bevacizumab as a potent inhibitor of inflammatory corneal angiogenesis and lymphangiogenesis. Invest Ophthalmol Vis Sci 48:2545-2552

12. Bock F, König Y, Kruse FE et al (2008) Bevacizumab $\left(\right.$ Avastin $^{\oplus}$ ) eye drops inhibit corneal neovascularisation. Graefes Arch Clin Exp Ophthalmol 246:281284

13. Bock F, Onderka J, Rummelt C et al (2009) Safety profile of topical VEGF-neutralization at the cornea. Invest Ophthalmol Vis Sci (Epub ahead of print)

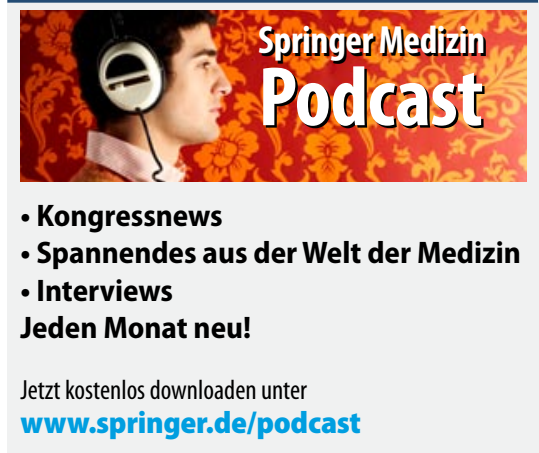

Jetzt kostenlos downloaden unter

www.springer.de/podcast 